\title{
Lixiviat de la décharge publique d'El Jadida (Maroc) : caractérisation et étude d'impact sur la nappe phréatique
}

\author{
CHOFQI Amina', YOUNSI Abdelkader', LHADI El Kbir', MANIA Jacky², MUDRY Jacques³ et VERON Alain ${ }^{4}$ \\ I. Faculté des sciences, BP 20, El Jadida, Maroc \\ 2. Eudil, Département géotechnique et génie civil , avenue Paul-Langevin, 59655 Villeneuve-d'Ascq Cedex, France \\ 3. Université de Franche-Comté, Département des géosciences, La Bouloie, 25030 Besançon Cedex, France \\ 4. Europôle méditerranéen de l'Arbois, BP 80, 13545 Aix-en-Provence Cedex 4, France
}

Pour tout contact : achofai@hotmail.com

\begin{abstract}
Résumé
Ce travail s'inscrit dans le cadre de l'étude d'impact sur l'environnement de la décharge municipale de la ville d'El Jadida, en particulier sur la nappe phréatique qui circule à faible profondeur ( 10 à $20 \mathrm{~m}$ avec un minimum de 0,9 m) sous un sol perméable.

L'analyse des lixiviats générés par cette décharge a montré qu'il s'agit d'un percolat à forte charge polluante minérale, organique et métallique (chlorures : $5680 \mathrm{mg} / \mathrm{l}$, sulfates : I $823 \mathrm{mg} / \mathrm{l}$, DCO : I $005 \mathrm{mg} / \mathrm{l}$, chrome : I $56 \mathrm{\mu g} / \mathrm{l}$ et cadmium : $34 \mu \mathrm{g} / \mathrm{l})$.

L'étude physico-chimique des eaux de puits de la région révèle une importante dégradation de la qualité de la nappe sous-jacente. En effet, des conductivités électriques (en moyenne de $8 \mathrm{mS} / \mathrm{cm}$ ) très corrélées à des teneurs élevées en chlorure (2 $000 \mathrm{mg} / \mathrm{l}$ ) et en sulfates ( I $200 \mathrm{mg} / \mathrm{l}$ ) sont fréquentes au niveau des puits, surtout de ceux situés à proximité et au niveau de la décharge. Ceci indique une forte contamination des eaux de ces puits par les lixiviats. Pour ce qui est de la charge organique et métallique (pratiquement négligeable dans les eaux souterraines naturelles), des teneurs anormalement élevées (210 mg/l en DCO, $21 \mathrm{mg} / \mathrm{l}$ en $\mathrm{DBO}_{5}, 20 \mu \mathrm{g} / \mathrm{l}$ en cadmium, $70 \mu \mathrm{g} / \mathrm{l}$ en chrome, $80 \mu \mathrm{g} / \mathrm{l}$ en nickel) ont été observées, principalement dans les puits situés au sein, à proximité et à l'aval de la décharge, dans le sens d'écoulement de la nappe.
\end{abstract}

\section{Introduction}

Si la mise en décharge demeure le moyen le plus économique et le plus utilisé pour l'élimination des déchets comparativement à d'autres modes, elle présente cependant des risques potentiels de dégradation de l'environnement par la production des biogaz et surtout des lixiviats qui véhiculent une importante charge polluante. Ces lixiviats, en s'infiltrant dans le sous-sol, entraînent une forte dégradation des eaux souterraines (Fedorak et al., 1987 ; Ozanne, 1990 ; Rivett et al., 1990 ; Grundfelt et Hoglund, 1995 ; Khattabi, 2002).
La décharge de la ville d'El Jadida est une décharge à ciel ouvert située à $2 \mathrm{~km}$ du centre ville, au milieu de zones résidentielles. D'une superficie de 40 hectares, elle est constituée de 3 carrières anciennement exploitées.

Opérationnelle depuis 1983, elle reçoit 140 tonnes/jours de déchets urbains (IICA, 1997) sous une forme mixte (domestiques, industriels et hospitaliers), et sans traitement préalable. Cette décharge non contrôlé et mal exploitée, génère un lixiviat non drainé et à forte charge polluante, qui s'infiltre à travers le sous-sol et contamine la nappe phréatique circulant à faible profondeur et qui remonte au fond des carrières.

Le présent travail a pour objectifs une caractérisation physicochimique du lixiviat et l'étude du suivi de la qualité des eaux prélevées dans les puits voisins, en considérant comme des indicateurs de pollution la conductivité électrique et les teneurs en chlorures et en matières organiques.

\section{Caractérisation du lixiviat de la décharge publique de la ville d'El Jadida}

Par lixiviat (appelé aussi percolat), on désigne l'eau qui a percolé à travers les déchets en se chargeant bactériologiquement et surtout chimiquement de substances minérales et organiques (Ozanne, 1990). Ces eaux peuvent contaminer les nappes phréatiques, les eaux superficielles et les sols si elles ne sont pas récupérées et traitées avant leur rejet dans le milieu naturel (Mejabri et al., 1995 ; Howard et al., 1996 ; Naifar, 1996 ; Amhoud, 1997 ; Jensen et al., 1999 ; Baun et al., 2000 ; Khattabi et al., 2002 ; Chofqi et al., 2004 ; Chofqi., 2004).

La composition des lixiviats est fonction de plusieurs paramètres, à savoir la nature et l'âge de la décharge, le type des déchets, la méthode d'enfouissement, la nature du site, les conditions climatiques... (Navarro et al., 1988 ; Leclerc et Bonneau, 1989 ; Blanchard et al., 1989 ; Matejka et al., 1994 ; Khattabi, 2002). 


\section{Echantillonnage et analyse}

La décharge de la ville d'El Jadida est dépourvue d'un système de drainage des lixiviats qui, après accumulation au fond dans la décharge, s'infiltrent à travers le sous-sol ou s'écoulent à travers les déchets pour aboutir au fond des carrières. A partir de ces fosses, le lixiviat a été prélevé et analysé deux fois par mois pendant la période allant de novembre 1999 à novembre 200 I. La caractérisation a porté sur la charge organique, minérale et métallique.

La température (T), la conductivité électrique (CE), le total des sels dissous (TDS) et le pH ont été mesurés in situ. Les concentrations des chlorures $\left(\mathrm{Cl}^{-}\right)$, sulfates $\left(\mathrm{SO}_{4}^{--}\right)$, calcium $\left(\mathrm{Ca}^{++}\right)$, magnésium $\left(\mathrm{Mg}^{++}\right)$, les bicarbonates $\left(\mathrm{HCO}_{3}{ }^{-}\right)$et la demande chimique en oxygène (DCO) ont été déterminés par volumétrie (AFNOR, 1987). Les concentrations en sodium $\left(\mathrm{Na}^{+}\right)$et en potassium $\left(\mathrm{K}^{+}\right)$ont été mesurées à l'aide d'un spectrophotomètre à flamme. La demande biologique en oxygène $\left(\mathrm{DBO}_{5}\right)$ a été mesurée par un DBO-mètre (WTW D 82362 Weilheim). L'azote total (NT) a été estimé selon la méthode de Kjeldhal (Kjeltec system 1002 Distilling unit). Les métaux lourds (chrome et cadmium) ont été mesurés à l'aide d'un spectrophotomètre d'absorption atomique (Unicam 929 AA Spectrometer). Les nitrates $\left(\mathrm{NO}_{3}{ }^{-}\right)$, l'ammonium $\left(\mathrm{NH}_{4}^{+}\right)$et les orthophosphates $\left(\mathrm{PO}_{4}{ }^{3-}\right)$ ont été déterminés par méthode calorimétrique à l'aide d'un spectrophotomètre. Les eaux de puits ont été aussi prélevées et analysées selon la même fréquence.

\section{Résultats}

Conductivité électrique et pH

Au cours du suivi bimensuel (1999-2000), les valeurs de la conductivité électrique enregistrées dans le percolat varient entre 16,8 et 38,2 mS/cm (Tableau I). La valeur moyenne annuelle de la conductivité électrique est de $26,45 \mathrm{mS} / \mathrm{cm}$. Les fortes valeurs sont observées en été : $\mathrm{CE}=37 \mathrm{mS} / \mathrm{cm}$ en juillet et $38,2 \mathrm{mS} / \mathrm{cm}$ en septembre (Tableau I). Par contre, c'est pendant la saison hivernale qu'on a obtenu les faibles conductivités $(16,8 \mathrm{mS} / \mathrm{cm}$ en janvier 2000 et 16,87 mS/cm en janvier 200 I). L'étude de la variation inter-annuelle de la conductivité électrique montre une légère augmentation de la CE de $26,45 \mathrm{mS} / \mathrm{cm}$ en 1999-2000 à 27, I 8 mS/cm en 2000-200। (Tableau 2).

Le lixiviat produit par la décharge d'El Jadida est très basique. Les valeurs du $\mathrm{pH}$ oscillent entre 8,4I et 9,3. La moyenne annuelle est de 8,76 (1999-2000) (Tableau I). Les valeurs maximales de $\mathrm{pH}$ sont enregistrées pendant l'été : $\mathrm{pH}=9,2$ en juillet et 9,3 en septembre (1999-2000) (Tableau I). Ces fortes valeurs coïncident avec les fortes températures enregistrées pour cette même période de l'année.

La variation inter-annuelle du $\mathrm{pH}$ (Tableau 2) témoigne d'une légère augmentation en fonction du vieillissement de la décharge : de 8,76 (1999-2000) à 8,84 (2000-200 I).

\begin{tabular}{|c|c|c|c|c|c|c|c|c|}
\hline Paramètre & Nov-99 & Jan-00 & Mars-00 & Mai-00 & Juill-00 & Sep-00 & Nov-00 & Moyenne \\
\hline $\mathrm{T}\left({ }^{\circ} \mathrm{C}\right)$ & 23 & 21,2 & 25,8 & 28,5 & 29,1 & 29,3 & 24 & 25,84 \\
\hline $\mathrm{pH}$ & 8,51 & 8,41 & 8,53 & 8,9 & 9,2 & 9,3 & 8,5 & 8,76 \\
\hline CE $(\mathrm{mS} / \mathrm{cm})$ & 16,51 & 16,8 & 18,2 & 34 & 37 & 38,2 & 18 & 26,45 \\
\hline $\operatorname{TDS}(\mathrm{g} / \mathrm{l})$ & 8,70 & 8,4 & 9,1 & 17 & 18,5 & 19,1 & 9 & 12,82 \\
\hline $\mathrm{Cl}^{-}(\mathrm{mg} / \mathrm{l})$ & 40001 & 3898,9 & 5001,2 & 6451,3 & 8002,5 & 9004,7 & 3394 & 5680 \\
\hline $\mathrm{Na}^{+}(\mathrm{mg} / \mathrm{l})$ & 1998 & 1930 & 2489 & 3190 & 3990 & 4489 & 1680 & 2823,71 \\
\hline $\mathrm{K}^{+}(\mathrm{mg} / \mathrm{l})$ & 840 & 780 & 1000 & 1200 & 2340 & 2440 & 1000 & 600 \\
\hline $\mathrm{Ca}^{++}(\mathrm{mg} / \mathrm{l})$ & 100,2 & 100 & 184,2 & 220,44 & 263,57 & 321,2 & 115,4 & 186,43 \\
\hline $\mathrm{Mg}^{++}(\mathrm{mg} / \mathrm{l})$ & 98,4 & 95,4 & 265,4 & 280,4 & 378,72 & 415,3 & 100 & 233,37 \\
\hline $\mathrm{SO}_{4}^{--}(\mathrm{mg} / \mathrm{l})$ & 1950 & 2000 & 1882 & 1780 & 1700 & 1654 & 1845 & 1823 \\
\hline $\mathrm{HCO}_{3}{ }^{-}(\mathrm{mg} / \mathrm{l})$ & 511,2 & 478,78 & 1450 & 2800 & 4392 & 5004 & 2400 & 2433,71 \\
\hline $\mathrm{NO}_{3}{ }^{-}(\mathrm{mg} / \mathrm{l})$ & 0,2 & 0,08 & 1,1 & 2,21 & 4,5 & 5 & 0,9 & 1,99 \\
\hline $\mathrm{NH}_{4}{ }^{+}(\mathrm{mg} / \mathrm{l})$ & 52 & 35 & 84 & 100 & 184 & 210 & 64 & 104,14 \\
\hline NTK (mg/l) & 98,4 & 70 & 64,34 & 145 & 210 & 290 & 94 & 138,32 \\
\hline $\mathrm{DBO}_{5}(\mathrm{mg} / \mathrm{l})$ & 88 & 94 & 62 & 61 & 42 & 28 & 90 & 66,43 \\
\hline DCO (mg/l) & 1350 & 1445 & 670 & 610 & 1000 & 1001 & 860 & 990,85 \\
\hline
\end{tabular}

Tableau I : Composition physico-chimique du lixiviat de la décharge d'El Jadida (1999- 2000).

\begin{tabular}{|c|c|c|}
\hline Paramètre & $\begin{array}{c}\text { Composition chimique } \\
\text { moyenne du lixiviat } \\
(1999-2000)\end{array}$ & $\begin{array}{l}\text { Composition chimique } \\
\text { moyenne du lixiviat } \\
(2000-2001)\end{array}$ \\
\hline $\mathbf{T}\left({ }^{\circ} \mathrm{C}\right)$ & 25,84 & 26,35 \\
\hline pH & 8,76 & 8,84 \\
\hline $\mathrm{CE}\left(\mathrm{mS} / \mathrm{cm}^{-1}\right)$ & 26,45 & 27,18 \\
\hline TDS (g/l) & 12,82 & 13,6 \\
\hline $\mathrm{Cl}^{-}(\mathrm{mg} / \mathrm{l})$ & 5680 & 5967 \\
\hline $\mathrm{Na}^{+}(\mathrm{mg} / \mathrm{l})$ & 2823,71 & 2941,66 \\
\hline $\mathrm{K}^{+}(\mathrm{mg} / \mathrm{l})$ & 600 & 700 \\
\hline $\mathrm{Ca}^{++}(\mathrm{mg} / \mathrm{l})$ & 186,43 & 202,83 \\
\hline $\operatorname{Mg}^{++}(\mathrm{mg} / \mathrm{l})$ & 233,37 & 255,83 \\
\hline $\mathrm{SO}_{4}^{-}(\mathrm{mg} / \mathrm{l})$ & 1823 & 1800 \\
\hline $\mathrm{HCO}_{3}{ }^{-}(\mathrm{mg} / \mathrm{l})$ & 2433,71 & 2743 \\
\hline $\mathrm{NO}_{3}{ }^{-}(\mathrm{mg} / \mathrm{l})$ & 1,99 & 2,37 \\
\hline $\mathrm{NH}_{4}{ }^{+}(\mathrm{mg} / \mathrm{l})$ & 104,14 & 114 \\
\hline NT $(\mathrm{mg} / \mathrm{l})$ & 138,32 & 146,16 \\
\hline $\mathrm{DBO}_{5}(\mathrm{mg} / \mathrm{l})$ & 66,43 & 55,33 \\
\hline DCO (mg/l) & 990,85 & 920 \\
\hline $\mathrm{DBO}_{5} / \mathrm{DCO}$ & 0,067 & 0,06 \\
\hline
\end{tabular}

Tableau 2 : Composition physico-chimique moyenne du lixiviat (1999-200I)

Les éléments inorganiques

Au cours de ce suivi, les teneurs en chlorures relevées dans le percolat montrent qu'il est très chargé en chlorures. Ces teneurs varient entre 3394 et 9004 mg/l (Tableau I), avec une moyenne annuelle de 5680 mg/l (1999 -2000).

L'étude de la variation de la concentration des chlorures a montré que cet élément présente une variation saisonnière très nette (Tableau I), avec des concentrations maximales pendant la saison estivale ( $8 \mathrm{~g} / \mathrm{l}$ en juillet et $9 \mathrm{~g} / \mathrm{l}$ en septembre [1999-2000]). Ces fortes concentrations coïncident avec les fortes valeurs de la conductivité électrique. Ceci indique que la conductivité est en grande partie déterminée par les ions chlorures. En hiver, nous assistons à une diminution de ces concentrations.

Le tableau I montre que la moyenne annuelle en sulfates est de | 820 mg/l (1999-2000), avec des concentrations qui oscillent entre 750 et | 450 mg/l. Contrairement aux chlorures, les valeurs maximales en sulfates sont enregistrées pendant la période hivernale : I 900 et $2000 \mathrm{mg} / \mathrm{l}$ respectivement en novembre et janvier (Figure I). 
Le lixiviat généré par la décharge est très riche en sodium, avec une teneur moyenne de $2820 \mathrm{mg} / \mathrm{l}$ en 1999-2000 et 2940 mg/l en 2000- 200I (Tableau I). Les concentrations maximales sont enregistrées pendant l'été. Le calcium et le magnésium présentent la même variation saisonnière que les chlorures et le sodium (teneurs maximales en été et des concentrations faibles en hiver [Tableau 2]). Ces valeurs restent toutefois faibles par rapport à celles de ces deux éléments. Les concentrations en bicarbonates sont relativement importantes. Elles oscillent entre 0,5 et 5 g/l (1999-2000) (Tableau I). En été, on enregistre une augmentation de la concentration de cet élément :4,4 et $5 \mathrm{~g} / \mathrm{l}$ respectivement en juillet et septembre (1999-2000). En hiver, on assiste à une diminution de ces teneurs (Tableau I).

\section{La matière organique}

Les concentrations en matière organique biodégradable $\left(\mathrm{DBO}_{5}\right)$ dans le lixiviat sont relativement faibles. En effet, elles varient entre 28 et $94 \mathrm{mg} / \mathrm{l}$ (Tableau I), avec une valeur moyenne annuelle de 66,43 mg/l (1999-2000). Les valeurs de la $\mathrm{DBO}_{5}$ diminuent pendant la période estivale : $42 \mathrm{mg} / \mathrm{l}$ en juillet et $28 \mathrm{mg} / \mathrm{l}$ en septembre ( 1999-2000). En hiver, on assiste à une augmentation de ce paramètre (Tableau I) : 90 mg/l en novembre et 94 mg/l en janvier (1999-2000).

Contrairement à la $\mathrm{DBO}_{5}$, les teneurs en matière organique oxydable (DCO) sont importantes, avec une moyenne annuelle égale à Ig/l (1999-2000). Ces concentrations varient entre 0,6 et I,4 g/l (1999-2000). Les valeurs maximales sont enregistrées en novembre et janvier ( I999-2000) : I,35 et I,45 g/l respectivement (Tableau I). A l'opposé de la $\mathrm{DBO}_{5}$, on constate une augmentation de ce paramètre pendant la période estivale (Tableau I). Le rapport $\mathrm{DBO}_{5} / \mathrm{DCO}$ est égal à 0,06 (2000-200 I).

\begin{tabular}{|l|c|c|c|}
\hline Paramètre & $\mathbf{1 9 9 6}^{*}$ & $\mathbf{1 9 9 7}^{*}$ & $\mathbf{2 0 0 0 - 2 0 0 1}^{*}$ \\
\hline pH & 8,3 & 8,5 & 8,84 \\
\hline DCO & 1900 & 1100 & 920 \\
\hline DBO $_{5}$ & 311 & - & 55,33 \\
\hline DBO $_{5} /$ DCO & 0,16 & - & 0,06 \\
\hline
\end{tabular}

Tableau 3 : Evolution inter-annuelle du pH, de la DCO, de la DBO et du rapport $\mathrm{DBO} / \mathrm{DCO}$.

\begin{tabular}{|c|c|c|c|c|}
\hline $\begin{array}{c}\text { Concentration } \\
(\mu \mathrm{g} / \mathrm{l})\end{array}$ & $\begin{array}{l}\text { Décharge } \\
\text { d'El Jadida } \\
\text { (Maroc) }\end{array}$ & $\begin{array}{c}\text { Décharge de } \\
\text { Rabat } \\
\text { (Maroc)* }\end{array}$ & $\begin{array}{l}\text { Décharge } \\
\text { d'Etueffont } \\
\text { (France)** }^{* *}\end{array}$ & $\begin{array}{l}\text { Fourchette de variation } \\
\text { d'une décharge } \\
\text { ménagère }{ }^{* \star *}\end{array}$ \\
\hline Zinc & 747,2 & - & 740 & $30-1.10^{6}$ \\
\hline Fer & 24000 & 23000 & 2630 & $3000-55.10^{6}$ \\
\hline Cuivre & 157,8 & 118 & 270 & $5-1.10^{4}$ \\
\hline Manganèse & 1256,72 & 4922 & - & $30-1,4.10^{6}$ \\
\hline Nickel & 133,8 & 133,6 & 210 & $15-1,3.10^{4}$ \\
\hline Chrome & 156,33 & 517 & 270 & $20-1500$ \\
\hline Cobalt & 208,6 & - & 60 & $5-1500$ \\
\hline Cadmium & 34 & 8,38 & 10 & - \\
\hline
\end{tabular}

Tableau 4 : Teneurs moyennes en métaux lourds du lixiviat de la décharge d'El Jadida (1999-200I) et comparatif des teneurs dans différents lixiviats.
L'étude de la variation inter-annuelle de la $\mathrm{DBO}_{5}$, de la $\mathrm{DCO}$ et du rapport $\mathrm{DBO}_{5} / \mathrm{DCO}$ témoigne d'une nette diminution de ces paramètres au cours du temps (Tableau 3). En effet, la $\mathrm{DBO}_{5}$ passe de $31 \mathrm{l}$ mg/l en 1996 à $55 \mathrm{mg} / \mathrm{l}$ (2000-200 I). La DCO, quant à elle, passe de 1900 mg/l ( I 996) à 920 mg // (2000-200 I). Le rapport $\mathrm{DBO}_{5} / \mathrm{DCO}$ diminue de 0, I6 en 1996 (Tazi, 2002) à 0,06 (2000-200 I)

Les éléments métalliques

L'examen des résultats présentés dans le tableau 4 montre clairement l'importante de la charge métallique. Le fer est le métal le plus abondant $(24 \mathrm{mg} / \mathrm{l})$. Le manganèse et le zinc présentent aussi des teneurs importantes qui sont respectivement de I,25 et 0,75 mg/l.

Les métaux toxiques présentent des teneurs relativement importantes : cobalt $=210 \mu \mathrm{g} / \mathrm{l} ;$ chrome $=156 \mu \mathrm{g} / \mathrm{l}$; nickel $=134 \mu \mathrm{g} / \mathrm{l}$.

La composition métallique du lixiviat issu de la décharge d'E Jadida est typique d'une décharge à caractère « ménager » dominant (Tableau 4). En effet, les concentrations des éléments métalliques du percolat étudié sont globalement identiques à celles des lixiviats générés par d'autres décharges d'ordures ménagères (Tableau 4), sauf pour certains éléments tels que le fer (décharge d'El Jadida $24 \mathrm{mg} / \mathrm{l}$, décharge d'Alger : 12,3 mg/l et décharge d'Etueffont : 2,6 mg/l).

\section{Discussion}

Les teneurs en matière organique du lixiviat produit par la décharge, exprimées en $\mathrm{DBO}_{5}$ et en $\mathrm{DCO}$, sont relativement faibles. La variation saisonnière de la $\mathrm{DBO}_{5}$ montre que c'est pendant la période estivale qu'elle présente les valeurs les plus faibles. Khattabi (2002) a montré qu'il y a une corrélation nette entre l'augmentation de la température et la diminution de la $\mathrm{DBO}_{5}$. En effet, pendant cette période, on note un réchauffement des eaux de lixiviation permettant ainsi une intensification de l'activité des bactéries. Celles-ci vont permettre la dégradation de la matière organique biodégradable présente dans le percolat. En hiver, les températures du jus de la décharge commencent à diminuer, constituant ainsi un facteur limitant de l'activité des bactéries, qui deviennent de plus en plus incapables de transformer cette matière organique biodégradable, ce qui conduit à une augmentation des valeurs de la $\mathrm{DBO}_{5}$ pendant cette période. A l'opposé de la $\mathrm{DBO}_{5}$, on note une augmentation de la DCO, probablement due aux produits des composés de biodégradation incomplète par les bactéries (Khattabi, 2002).

Le calcul du rapport $\mathrm{DBO}_{5} / \mathrm{DCO}$ nous a permis de caractériser l'âge et l'état de la décomposition des déchets. II faut rappeler que $\mathrm{DBO}_{5} / \mathrm{DCO}$ est un rapport qui évolue dans le temps. II indique le degré de biodégradabilité et donne des informations sur la nature des transformations biochimiques qui règnent au sein de la décharge (Kerbachi 
et Belkacemi, 1994). Ainsi, pour les décharges jeunes où l'activité biologique correspond à la phase acide de dégradation anaéobie, ce rapport atteint la valeur de 0,83. A l'opposé, pour les anciennes et vieilles décharges, ce rapport décroît jusqu'à 0,05. Pour ces décharges, l'étape de fermentation ultime est atteinte. Ce stade de fermentation anaérobie est caractérisé par la diminution de la DCO et surtout de la $\mathrm{DBO}_{5}$, avec un pH basique (Ozanne, 1990 ; Robinson, 1991 ; Amokrane, 1994 et Desachy, 1994). La DBO 5 peut même devenir nulle au cours des années. Cette phase est aussi caractérisée par des teneurs importantes en ammonium. Dans notre cas, il semble que la décharge d'El Jadida est une décharge vieille qui traverse actuellement le stade de la méthanogénèse $\left(\mathrm{DBO}_{5} / \mathrm{DCO}\right.$ égal à 0,06$)$. Le $\mathrm{pH}$ basique (8,84 en moyenne), mesuré sur ces lixiviats, reflète bien cette phase d'évolution biologique. Ceci permet d'expliquer les faibles valeurs trouvées de la $\mathrm{DBO}_{5}$, qui continuent à diminuer au cours du temps. Nous pouvons conclure que le lixiviat de la décharge d'El Jadida est un lixiviat vieux et stabilisé, caractérisé par une biodégradabilité moindre et une charge organique complexe.

La diminution de la $\mathrm{DBO}_{5}$ à la suite de la biodégradation a entraîné une production relativement importante en azote total et en ammonium. Les teneurs en nitrates dans le lixiviat restent cependant très faibles, voire même nulles suite aux phénomènes de réduction qui règnent dans le site de la décharge.

Le lixiviat présente des valeurs de conductivité et des teneurs en chlorures très importantes. II s'agit d'un caractère commun à toutes les décharges d'ordures ménagères (Ozanne, 1990 ; Kerbachi et Belkacemi, 1994 ; Amhoud, 1997, Christensen et al., 200 I et Khattabi, 2002). Les fortes teneurs en chlorures sont enregistrées pendant la période estivale et coïncident avec les maxima des températures. Ceci peut s'expliquer d'une part par le fait que pendant cette période de l'année (l'été), il y a une absence quasi-totale des précipitations qui peuvent engendrer des phénomènes de dilution du percolat, d'où le fait que les lixiviat deviennent de plus en plus riches en chlorures. D'autre part, l'augmentation de la température semble stimuler les réactions d'oxydation, d'hydrolyse et de reminéralisation des déchets par les bactéries (stimulation de l'activité enzymatique bactérienne), d'où le fait que le lixiviat devient riche en éléments minéraux tels que les chlorures (Mattias et Nilson, 1997 ; Khattabi, 2002 ;Tazi, 2002). L'évolution des concentrations en chlorures semble influencer celle de la conductivité électrique, qui est déterminée principalement par ces ions. En effet, ces concentrations présentent les mêmes variations saisonnières, annuelles et inter-annuelles. Cette relation étroite entre les teneurs en chlorures et la conductivité a été démontrée par Khattabi (2002) sur le site d'Etueffont.

Les plus faibles teneurs en sulfates sont enregistrées en été, saison pendant laquelle les phénomènes de biodégradation bactérienne sont très intenses (réchauffement du lixiviat) et les conditions du milieu très réductrices. Les sulfates sont ensuite réduits en sulfures $\left(\mathrm{H}_{2} \mathrm{~S}\right)$ qui sont parmi les gaz responsables des mauvaises odeurs dégagées par la décharge (Khattabi, 2002 ;Tazi, 2002).

Comme pour la plupart des éléments inorganiques, l'évolution des concentrations en calcium, magnésium et en sodium dans le lixiviat est en relation avec la teneur en substance organique dissoute (Christensen et al., 200 I). En effet, les concentrations maximales de ces trois éléments sont enregistrées pendant la période estivale. Ceci peut être dû au fait que pendant cette période, la teneur en matière organique qui est responsable de la complexation de ces éléments est très faible.

L'analyse de la composition en métaux lourds du lixiviat a mis en évidence l'importante charge métallique de ce percolat, bien qu'il s'agisse d'une composition typique d'une décharge à caractère ménager dominant.

Les concentrations en manganèse et surtout en fer sont anormalement élevées dans le percolat, ce qui n'est habituellement pas le cas pour un lixiviat généré par une décharge en phase de méthanisation (cas étudié). Ceci est vraisemblablement dû au fait que la décharge continue toujours à recevoir des déchets renfermant du fer, surtout les déchets industriels qui y sont rejetés à l'état brut et mélangés aux déchets ménagers.

Bien que la composition métallique du lixiviat soit typique d'une décharge à caractère ménager dominant et que cette charge en métaux lourds reste cependant faible par rapport à celle des lixiviats des décharges d'ordures essentiellement industrielles, la charge métallique présente toutefois un risque énorme pour les eaux souterraines du fait qu'il y a un contact direct du lixiviat avec la nappe sous-jacente au niveau des carrières de la décharge. D'autre part, la faible profondeur des eaux souterraines circulant dans la zone de la décharge, la perméabilité de la zone non saturée et l'état dégradé et saturé des sols sablo-argileux du site de la décharge favorisent les phénomènes d'infiltration et de percolation des lixiviats et de leur charge métallique. Dans notre cas, les phénomènes d'épuration et de complexation par la zone non saturée et le sol de la décharge n'ont pas un rôle significatif.

\section{Evaluation de l'impact du lixiviat sur les eaux souterraines}

Le site de la décharge d'El Jadida est un site perméable (perméabilité de fissure). II est formé essentiellement par des calcaires et des marno-calcaires d'âge cénomanien. En absence d'un système de drainage, les lixiviats générés s'infiltrent à travers le sous-sol pour atteindre la nappe phréatique sous-jacente circulant à très faible profondeur ( 10 à $20 \mathrm{~m}$ ) avec un minimum de $0,9 \mathrm{~m}$ et qui affleure au fond des carrières au sein de la décharge. Le sens de l'écoulement de la nappe se fait de la décharge vers l'océan, ce qui entraine la propagation du flux de pollution vers les puits se trouvant en aval. 


\section{Conductivité électrique}

D'aprés la figure I, l'ensemble des puits suivis pendant cette étude présentent des conductivités électriques importantes (moyenne annuelle d'environ 4,50 mS/cm), supérieures aux normes françaises de potabilité (1,5 $\mathrm{ms} / \mathrm{cm})$. L'analyse de la carte de distribution spatiale de la conductivité (Figure I) a permis de montrer un resserrement très marqué des courbes d'isoconductivité au centre, aux alentours et dans la partie avale proche du site de la décharge, ceci traduisant des valeurs importantes de la conductivité et donc une salinité très élevée au niveau des puits situés dans ces secteurs sensibles. En effet, au niveau de ces points d'eau, la conductivité moyenne varie entre 8,88 et $8,95 \mathrm{mS} / \mathrm{cm}$ et peut même dépasser $9,5 \mathrm{mS} / \mathrm{cm}$ par endroit et par saison. Cette forte salinité ne peut être due qu'à une contamination par les lixiviats générés au niveau de la décharge (Khattabi, 2002 ;Tazi, 2002 ; Chofqi et al., 2004). En effet et comme pour l'ensemble des percolats de décharge incontrôlée, nous avons pu démontrer précédemment que les lixiviats de la décharge d'El Jadida, sont caractérisées par des fortes valeurs de conductivité électrique pouvant atteindre des moyennes annuelles de l'ordre de $26,45 \mathrm{mS} / \mathrm{cm}$.

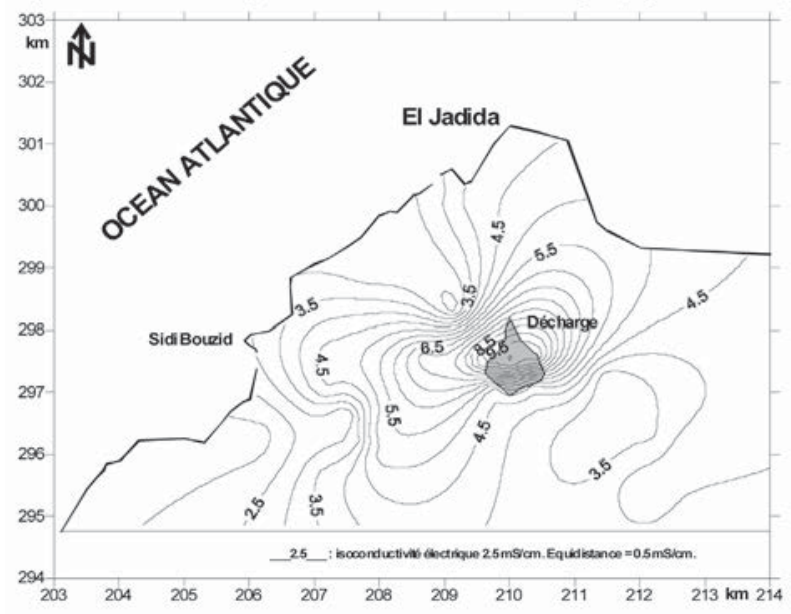

Figure I : Distribution spatiale de la conductivité électrique (septembre 200I).

Cette pollution des eaux souterraines au voisinage du site de la décharge se fait selon deux mécanismes :

- une contamination directe au niveau des trois carrières de la décharge et qui est due au contact des lixiviats avec la nappe qui remonte au fond des carrières ;

- une contamination indirecte due à l'infiltration et à la percolation des lixiviats à travers le sol et la zone non saturée, en général perméables. Elle est favorisée par la faible profondeur de la nappe sous-jacente (10 à $20 \mathrm{~m}$ ). Cette pollution va ensuite s'étaler dans la même direction que celle de l'écoulement de la nappe, pour atteindre les puits situés en aval de la décharge. Ce qui explique les fortes valeurs de conductivité enregistrées au niveau de ces puits. Nous pouvons conclure que la minéralisation totale des eaux souterraines circulant à proximité immédiate et à l'aval du site de la décharge étudiée est déterminée par celle des lixiviats entrant en contact direct et indirect avec la nappe.

\section{Les chlorures}

Les résultats obtenus montrent que les teneurs en chlorures sont très importantes (moyenne annuelle $=1050 \mathrm{mg} / \mathrm{l}$ ) et dépassent largement les normes de potabilité (250 $\mathrm{mg} / \mathrm{l}$ ) et les limites supérieures admissibles (600 mg/l) fixées par l'OMS (OMS, 1984).

La carte des chlorures (Figure 2) montre toujours une distribution spatiale similaire à celle de la conductivité électrique. Elle est toujours caractérisée par des zones de concentrations élevées dans les secteurs situés aux alentours et à l'aval de la décharge.

Cette forte charge en ions chlorures (en moyenne de 2 $000 \mathrm{mg} / \mathrm{l}$ ) est liée à une contamination directe et indirecte par les lixiviats qui sont fortement chargés en chlorure (5 $680 \mathrm{mg} / \mathrm{l})$. En effet, les déchets renferment des teneurs importantes en chlorures (Reitzel, 1990).

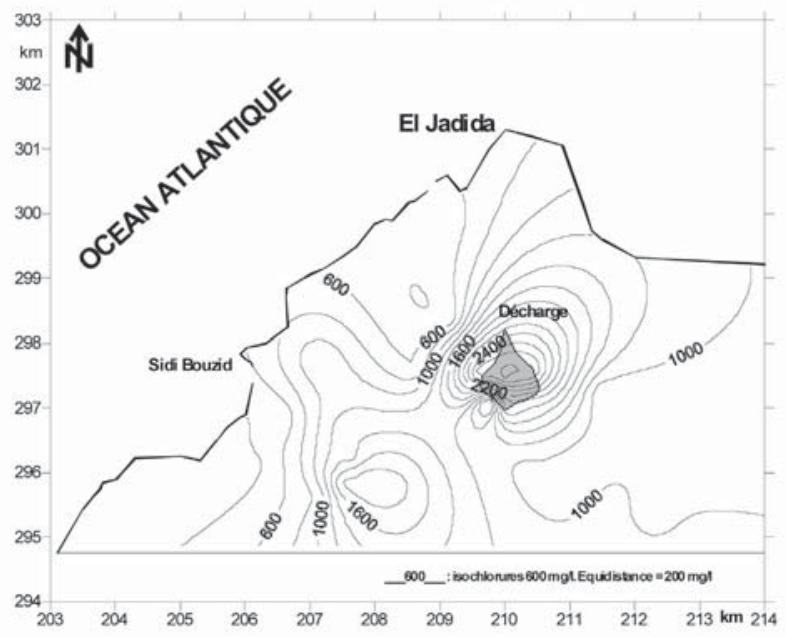

Figure 2 : Distribution spatiale des chlorures (septembre 200I).

La dégradation de ces déchets au niveau des décharges produit des percolats très chargés en cet élément chimique (Ozanne, 1990). L'ion chlorure est un élément très mobile, qui migre facilement vers les nappes sous-jacentes. II n'est pas affecté par les phénomènes d'adsorption ou d'échanges d'ions, il n'intervient pas dans les équilibres acido-basiques ou d'oxydoréduction et il n'est pas retenu par les complexes argilo-humiques des sols. C'est pourquoi il est fréquemment utilisé comme un bon traceur conservatif qui permet de mettre en évidence l'impact des lixiviats sur la qualité physicochimique des nappes phréatiques (Appelo et Potsma 
1993 ; Khattabi et al., 2001 ; Khattabi, 2002). La contamination des eaux de la nappe par les lixiviats prend l'aspect d'un panache de pollution qui s'étale tout au tour du site de la décharge et atteint les puits situés dans les secteurs aval, en concordance avec le sens d'écoulement de la nappe. Ce qui explique les fortes teneurs en chlorures enregistrées au niveau de ces puits.

\section{La matière organique}

Les eaux souterraines naturelles contiennent généralement peu ou pas de matière organique (Guessir, 1995). Cependant, les puits $\mathrm{P}|7, \mathrm{P}| 8$ et $\mathrm{P} \mid 9$, situés à proximité directe de la décharge (Figure 3), présentent des teneurs en DCO très élevées, avec des moyennes annuelles de 208 à 21 I mg/l et peuvent même dépasser 300 mg/l. Ces teneurs sont proches de celle des eaux usées marocaines (Tazi, 2002). Ce qui montre l'acuité des problèmes de contamination organique de ces puits par les lixiviats de la décharge. Les valeurs de la $\mathrm{DBO}_{5}$ sont aussi importantes (les moyennes annuelles varient entre 20,6 et 22,9 I mg/l) et peuvent même dépasser $40 \mathrm{mg} / \mathrm{l}$.

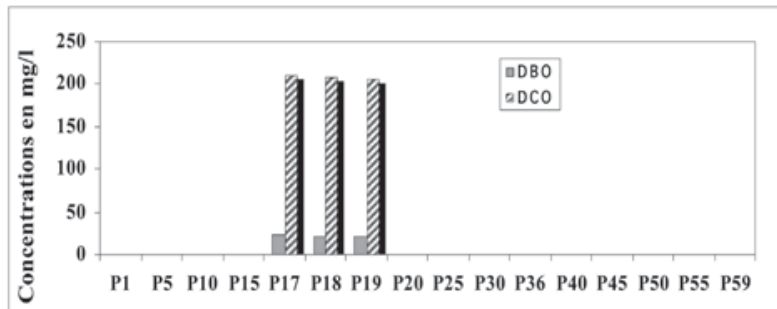

Puits

Figure 3 : Teneur en DBO et en DCO dans les puits étudiés.

Cette forte charge organique montre qu'au niveau de cette zone, la nappe souterraine reçoit un flux important de matière organique qui provient de la dégradation de déchets au niveau de la décharge et qui percole à travers les eaux de lixiviation.

\section{Conclusion}

Au terme de cette étude on peut dire que :

- Après 20 ans d'exploitation, la décharge de la ville d'El Jadida traverse actuellement l'étape d'activité biologique correspondant à la phase méthanique de dégradation anaérobie : méthanogenèse $\left(\mathrm{DBO}_{5} / \mathrm{DCO}: 0,056\right)$;

- Les lixiviats produits par la décharge de la ville entrent dans la catégorie des lixiviats vieux stabilisés à forte charge polluante non ou faiblement biodégradable. Ces percolats sont d'une importante charge métallique avec des teneurs très élevées en métaux;
- En absence d'un système de récupération des lixiviats, et en présence d'un sol perméable et d'une nappe circulant à de faible profondeur, ces percolats, après leur génération, s'accumulent au fond des déchets et s'infiltrent à travers le sol pour atteindre la nappe sous-jacente, entraînant ainsi sa contamination et la dégradation de sa qualité physicochimique ;

- La remonté de la nappe au fond des carrières de la décharge favorise cette contamination suite à un contact direct avec les lixiviats ;

- L'analyse physico-chimique des eaux des puits de la région révèle une forte dégradation de ces eaux suite à leur contamination par les eaux de percolation générées par la décharge. II s'agit d'une pollution à la fois physique (conductivité électrique élevée) et chimique (forte salinité et concentrations élevées en matière organique) ;

- L'étude de la répartition spatiale des différents contaminants a montré l'existence d'un panache de pollution aux alentours de la décharge et qui a tendance à s'étaler dans le sens d'écoulement de la nappe.

\section{Bibliographie}

AFNOR 1989, Eau, méthodes d'essai, Association française de normalisation. Edition 1989, Paris.

JICA 1997, Projet de site de décharge pour El Jadida. Rapport d'avancement (2), 3, 49 p.

Amhoud S. 1997, Apports de la géologie et de l'hydrogéologie à l'étude de l'impact de la décharge d'Oued Akrech sur les ressources en eau, Thèse de $3^{\text {e }}$ cycle, Faculté des sciences de Rabat.

Appelo C.A.J., Potsma D. 1993, Geochemistry, groundwater and pollution. A.A. Balkema, Rotterdam, 540 p.

Baun A., Jensen, S.D., Bjerg L., Christensen T.H., Nyholm N. 2000, Toxicity of organic chemical pollution in groundwater down gradient of a landfill (Grindsted, Denmark). Environmental and technology, 34, 647-I652.

Chofqi A. 2004, Mise en évidence des mécanismes de contamination des eaux souterraines par les lixiviats d'une décharge incontrôlée (El Jadida, Maroc) : géologie, hydrogéologie, géoélectrique, géochimie et épidémiologie. Thèse, Université d'El Jadida, Maroc, 250 p.

Christensen T H., Kjeidsen R., Bjerg P L., Jensen D L., Christensen J B., Bauna A., Albrechtsen H J., Heron G. 200I, Biogeochemistry of landfill leachate plumes. Appl. Geochemistry, 16, 659-7I8.

Desachy C. 1994, Les déchets en 1994.Techniques, Sciences et Méthodes - L'Eau, I I, 603-609.

Fedorak P. M., Hrudey S.E. 1987, Water Science and Technology, 19, 219 228.

Grundfelt B., Hoglund L.O. 1995, Some views on effect of different types of barriers in waste deposits Groundwater Quality. Rémédiation and Protection. In Proceeding of the Prague conference. International Association of Hydrological Sciences (IAHS), 347-354. Publication No 225.

Guessir H. 1995, Etude de l'impact de l'irrigation par les eaux usées brutes sur la qualité physico-chimique du sol et de la nappe phréatique dans la région de Sidi Bennour (Maroc). Thése de $3^{e}$ cycle, Université Chouaib Doukkali, El Jadida, I 34 p. 
Howard Ken W.F., Eyles N., Liyingstone S. 1996, Municipal landfill practice and its impact on groundwater resources in and around urban Toronto, Canada. Hydrog. J, 4, 64-79.

Kerbachi R., Belkacemi M. 1994, Caractérisation et évolution des lixiviats de la décharge de Oued-Smar à Alger.Techniques, Sciences et Méthodes - L'Eau, II, 615-6|8.

Khattabi H. 2002, Intérêts de l'étude des paramètres hydrogéologiques et hydrobiologiques pour la compréhension du fonctionnement de la station de traitement des lixiviats de la décharge d'ordures ménagères d'Etueffont (Belfort, France). Thése, Université de Franche-Comté, France.

Khattabi H., Lotfi A., Mania J. 200I, Evolution temporelle de la composition du lixiviat d'une décharge à ciel ouvert : effet des précipitations. Déchets, Sciences et Techniques, 21, 7-10.

Khattabi H., Lotfi A., Mania J. 2002, Evaluation de l'impact des lixiviats d'une décharge d'ordures ménagères sur la qualité physico-chimique et bactériologique des eaux d'un ruisseau de Franche-Comté. Déchets, Sciences et Techniques, 24, I- 4.

Leclerc G., Bonneau A. 1989, La lixiviation : source, composition et atténuation. Eau du Québec, I5 (I), 37-45.

Matejka G., Rinke M., Mejbri R., Bril H. 1994, Pollution engendrée par une décharge d'ordures ménagères: Bilan hydrique et caractérisation. Environmental Technology, I5, 313-322.

Mattias B.A., Nilsson P. 1997, Seasonal changes of leachate production and quality from test cells. J. Envir. Eng. Div. Assce, 892-900.

Mejbri R., Matejka G., Lafrance P., Mazet M. 1995, Fractionnement et caractérisation de la matiére organique des lixiviats de décharge d'ordures ménagères. Sci. Eau, 8, 217-236.

Naifar E.M. 1996, La situation actuelle de l'élimination des ordures ménagères de l'agglomération de Rabat. Etude de l'impact des rejets liquides de la décharge d'Akrach sur l'environnement, Thèse de $3^{\text {e }}$ cycle, Faculté des sciences, Rabat.

Navarro A., Bernard D., Millot N. 1988, Les problèmes de pollution par les lixiviats de décharge. Techniques, Sciences et Méthodes - L'Eau, 3, 54 I-545.

OMS. 1993, Guidelines for Drinking Water Quality, Geneva, Switzerland.

Ozanne F. 1990, Les lixiviats de décharge, le point des connaissances en 1990. Techniques, Sciences et Méthodes - L'Eau, juin 1990, 289-3I2.

Reitzel A. 1990, The temporal characterization of municipal solid waste leachate. Waterloo, Ontario, Univ. Waterloo, Masters of applied Science Thesis, $144 \mathrm{p}$

Rivett M.O., Lerner D.N., Liyod J.W. 1990, Chlorinated solvents in UK Aquifers. Journal of Water and Management, 4, 242-250.

Robinson H D. 1991, Characterisation and treatment of leachates from Hong Kong Landfill Sites. J, IWEM, 5, 326-335.

Tazi H. 200I, Déchets solides : étude d'impact sur l'environnement (sol, eaux souterraines) et traitement par voie de compostage. Thèse, Université d'El Jadida, Maroc, 224 p. 